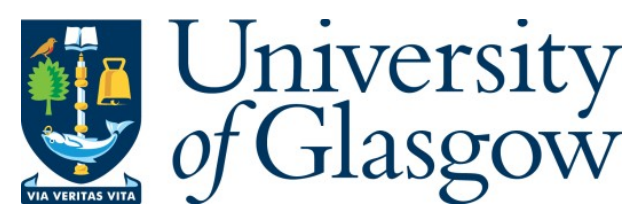

Liang, K., and Ruess, M. (2016) Nonlinear buckling analysis of the conical and cylindrical shells using the SGL strain based reduced order model and the PHC method. Aerospace Science and Technology, 55, pp. 103-110.

There may be differences between this version and the published version. You are advised to consult the publisher's version if you wish to cite from it.

http://eprints.gla.ac.uk/120002/

Deposited on: 09 June 2016

Enlighten - Research publications by members of the University of Glasgow http://eprints.gla.ac.uk 


\title{
Nonlinear buckling analysis of the conical and cylindrical shells using the SGL strain based reduced order model and the PHC method
}

\author{
Ke Liang ${ }^{\mathrm{a}, *}$, Martin Ruess ${ }^{\mathrm{b}}$ \\ ${ }^{a}$ Qian Xuesen Laboratory of Space Technology, China Academy of Space Technology, Beijing 100094, China \\ ${ }^{b}$ School of Engineering, University of Glasgow, Rankine Building, Oakfield Avenue G12 8LT, United Kingdom
}

\begin{abstract}
Thin-walled conical and cylindrical shells subjected to axial compression often show a snap-back response in the presence of buckling. Newton iterations based path-following methods cannot trace reliably the snap-back response due to the extremely sharp turning angle near the limit point, and the original Koiter-Newton method also meets difficulties to achieve a complete post-buckling response beyond the limit point. In this paper, the improved KoiterNewton method is proposed to trace the post-buckling path of cylinders and cones, in the framework of the reducedorder modeling technique. The polynomial homotopy continuation (PHC) method is used to solve the lower-order nonlinear reduced order model reliably and efficiently. The simplified Green-Lagrange (SGL) kinematics which consider the stress redistribution after buckling are implemented into the construction of the reduced order model to produce accurate results for curved shells. The numerical results presented reveal that the improved method is a robust and efficient technology to achieve the entire nonlinear response for the snap-back case.
\end{abstract}

Keywords: Buckling, Snap-back, PHC method, Simplified Green-Lagrange kinematics, Reduced order model

\section{Introduction}

Thin-walled conical and cylindrical shells are commonly used as primary components in weight-critical structure engineering, such as aircrafts and rockets, due to the high specific strength and stiffness $[1,2,3]$. Their load-carrying capabilities are often determined by the buckling loads which may be much lower than the failure loads of materials. These shell type structures, which exhibit an unstable post-buckling behavior, are highly sensitive to the initial imperfections, especially to the geometric imperfection [4].

Nonlinear structural analysis based on a path-following technique is commonly used to trace the response curve and to predict the load-carrying capacity of shell structures in the presence of buckling [2,5]. Snap-through and snap-back responses are two main phenomenons usually associated with the buckling of shell structures [6]. Some variants of the classical Newton method, i.e. the arc-length method [7] and norm flow method [8], have been proved to deal with the snap-through case very well. However, the above methods encounter difficulties with a snap-back response of cylindrical shells [9], where extremely sharp turning angles are present [3, 5, 10]. A significant reduction of the incremental step size is required to distinguish properly the two closely spaced path segments near the limit point $[5,11]$. When instabilities are localized, there will be a local transfer of strain energy from one part of the model to neighboring parts which may prevent the success of global solution methods. This class of problems must be solved either dynamically or with the aid of artificial damping. Thus, a combination of the displacement control and a damping factor is commonly used to pass the limit point of a snap-back response [5, 10].

The Koiter-Newton $(\mathrm{KN})$ method $[12,13]$ has been proposed based on the reduced-order modeling technique to trace the nonlinear equilibrium path in a stepwise manner. In each step, the method combines a prediction phase using a nonlinear reduced order model(ROM) based on Koiter asymptotic analysis $[14,15,16]$ with a Newton iteration based correction procedure, thus allowing the algorithm to use fairly large step sizes. In the original Koiter-Newton

${ }^{*}$ Corresponding Author. Tel.: +86 01068 113099; fax: +86 01068 113099. E-mail address: liangke.nwpu@ 163.com; liangke@qxslab.cn 
method, the classical arc-length method is used to solve the nonlinear system of equations associated with the reduced order model. Instead of solving a large-scale nonlinear system generated from the full finite element model [17], the lower-order reduced order model can provide a much more reliable solution to pass the extremely sharp turning angle in the snap-back case. However, the path-following performance is still very sensitive to the solution parameters related to the arc-length method and the method meets difficulties to trace the post-buckling path that is far beyond the limit point. Actually, the nonlinear algebraic equations, that is the reduced order model, can be expressed as polynomial equations. Thus, the polynomial homotopy continuation (PHC) method [18, 19] can be used as a reliable and efficient tool to solve the lower-order reduced order model. The construction of the reduced order model requires derivatives of the strain energy with respect to the degrees of freedom up to the fourth order, which is two orders more than traditionally needed for a Newton based nonlinear finite element technique. The von Kármán kinematics have been used in the finite element implementation of the original Koiter-Newton method [13]. The nonlinear in-plane rotation terms are neglected to facilitate the high order derivatives of the strain energy. However, these terms might be negligible for flat plate situations but they indeed play a major role in structures consisting of assembly of flat plates or curved shells. A alternative way to alleviate the shortcoming is to use the simplified Green-Lagrange (SGL) strain tensor which has been successfully implemented in the former Koiter reduction method [16].

The contribution of this paper distinguishes significantly from previous publications $[6,12,13,20]$ in the improvement of the original Koiter-Newton method to be more applicable for the snap-back case. The lower-order reduced order model is solved using the polynomial homotopy continuation method, to trace reliably the entire snap-back response. The simplified Green-Lagrange kinematics are implemented into the Koiter-Newton method to obtain a more accurate results for the curved shells. We carefully test the improved Koiter-Newton method for the snap-back behaviour of thin-walled cylindrical and conical shells to reveal the performance of the method for extremely sharp turning angles at the limit point. We demonstrate that the method is capable to handle these numerically severe test cases reliably and accurate and thus outperforms most of the state-of-the-art solution methods.

The rest of the paper is organized as follows: a brief introduction of the shell theory and the SGL strain used in this study is given in section 2. The Koiter-Newton method and the PHC method used to solve the reduced order model are presented in section 3. Numerical examples of cylinders and cones used to demonstrate the success of the method are provided in section 4 . We summarize the paper and draw conclusions in section 5 .

\section{Shell theory based on simplified SGL strain}

Based on the classical plate theory (Kirchhoff-Love hypothesis), the three displacement components $(u, v, w)$ of a thin plate are expressed as:

$$
\left\{\begin{array}{l}
u(x, y, z)=u^{0}(x, y)-z \frac{\partial w}{\partial x} \\
v(x, y, z)=v^{0}(x, y)-z \frac{\partial w}{\partial y} \\
w(x, y)=w^{0}(x, y)
\end{array}\right.
$$

where $u^{0}(x, y), v^{0}(x, y)$ and $w^{0}(x, y)$ are the displacement components related to the mid-plane of the plate.

The total strain vector $\varepsilon$ for the plate is:

$$
\boldsymbol{\varepsilon}=\boldsymbol{\varepsilon}_{m}+z \boldsymbol{\kappa},
$$

where $\boldsymbol{\varepsilon}_{m}$ and $\boldsymbol{\kappa}$ are the in-plane strain vector of the mid-plane and the curvature vector of the plane.

To consider the geometrical nonlinearities of the plate, the simplified Green-Lagrange strain kinematics are used for the mid-plane strain $\boldsymbol{\varepsilon}_{m}$, as given by:

$$
\boldsymbol{\varepsilon}_{m}=\left\{\begin{array}{lll}
\varepsilon_{x} & \varepsilon_{y} & \varepsilon_{x y}
\end{array}\right\}
$$


where

$$
\left\{\begin{array}{l}
\varepsilon_{x}=\frac{\partial u}{\partial x}+\frac{1}{2}\left(\frac{\partial v^{2}}{\partial x}+\frac{\partial w^{2}}{\partial x}\right) \\
\varepsilon_{y}=\frac{\partial v}{\partial y}+\frac{1}{2}\left(\frac{\partial u^{2}}{\partial y}+\frac{\partial w^{2}}{\partial y}\right) \\
\varepsilon_{x y}=\frac{1}{2}\left(\frac{\partial u}{\partial y}+\frac{\partial v}{\partial x}\right)+\frac{1}{2}\left(\frac{\partial w}{\partial x} \frac{\partial w}{\partial y}\right)
\end{array},\right.
$$

The simplified Green-Lagrange strain kinematics include some nonlinear in-plane rotation terms, which consider the stress redistribution in the post-buckling deformation.

The constitutive relationship of the plate is written as:

$$
\left\{\begin{array}{c}
\mathbf{N} \\
\mathbf{M}
\end{array}\right\}=\left[\begin{array}{ll}
\mathbf{A} & \mathbf{B} \\
\mathbf{B} & \mathbf{D}
\end{array}\right]\left\{\begin{array}{c}
\boldsymbol{\varepsilon}^{m} \\
\boldsymbol{\kappa}
\end{array}\right\}
$$

where $\mathbf{N}$ and $\mathbf{M}$ are the membrane force vector and the bending moment vector, respectively, and matrices $\mathrm{A}, \mathrm{B}$ and $\mathrm{D}$ are the membrane stiffness, membrane-bending coupling stiffness and bending stiffness, respectively. For the isotropic shell, the coupling stiffness $B$ equals to be 0 . For laminated composite plate, the material stiffness $A, B$ and $D$ can be calculated using the classical lamination theory.

\section{The improved Koiter-Newton method}

In the following we briefly present the basic ideas and principles of the Koiter-Newton method. In particular, we introduce the polynomial homotopy continuation method used to solve the reduced order model. For a detailed description of the theory, we point the reader to work $[12,13,18]$.

\subsection{Construction of the reduced order model}

The Koiter-Newton method is based on a step by step procedure to trace the equilibrium path of the deforming structure, that is similar to classical path-following techniques [21]. The unique properties and algorithmic differences compared to standard technologies are given in the following with the help of Figs. 1 and 2. In addition, the red contents written in Fig. 1 are the main improvements made in this study, compared to the original KN method.

Starting from a known nominal equilibrium state $\left(\mathbf{q}_{0}, \lambda_{0}\right)$, usually the un-deformed configuration of a structure, the equilibrium state is represented by a set of nonlinear algebraic equations:

$$
\mathbf{f}_{\text {int }}(\mathbf{q})=\lambda \mathbf{f}_{\text {ext }}
$$

where $\mathbf{f}_{i n t}$ and $\mathbf{f}_{e x t}$ are the internal force vector and external force vector, respectively, and $\lambda$ is the load parameter, and $\mathbf{q}$ is the displacement vector.

The corresponding reduced order model at this known state is constructed within the framework of the KoiterNewton method, to be:

$$
\overline{\mathcal{L}}(\xi)+\bar{Q}(\xi, \xi)+\bar{C}(\xi, \xi, \xi)=\phi
$$

where $\overline{\mathcal{L}}, \bar{Q}$ and $\bar{C}$ are still to be determined linear, quadratic and cubic forms of the approximated load amplitudes $\phi$. These forms can also be represented by a two-dimensional tensor $\bar{L}$, a three-dimensional tensor $\bar{Q}$ and a fourdimensional tensor $\overline{\mathrm{C}}$ of order $(1+m)$, respectively, where $m$ is the number of the closely-spaced buckling modes of the structure. $\boldsymbol{\xi}$ are generalized displacements or perturbation parameters.

The construction of the reduced order model (7) is illustrated in Fig. 1. The basic idea involves a modification of Koiter's asymptotic theory to make it applicable already from the unloaded state. The unknowns $\overline{\mathcal{L}}, \bar{Q}$ and $\bar{C}$ in the reduced order model (7) can be achieved by solving three sets of linear equations. The number of degrees of freedom in the reduced order model is $m+1$, where $m \ll N$ and where $N$ is the total number of DOFs of the full discretized model. In this study, the simplified Green-Lagrange strain kinematics introduced in section 2 are used to construct the 
reduced order model to properly consider the stress redistribution after buckling, which is an important improvement compared to the original $\mathrm{KN}$ method.

The structural imperfection is introduced easily in the Koiter-Newton method. The construction of the reduced order model remains unchanged when imperfections are introduced or changed during the analysis, which reduces the computational cost significantly for buckling analyses when imperfection models are tested systematically.

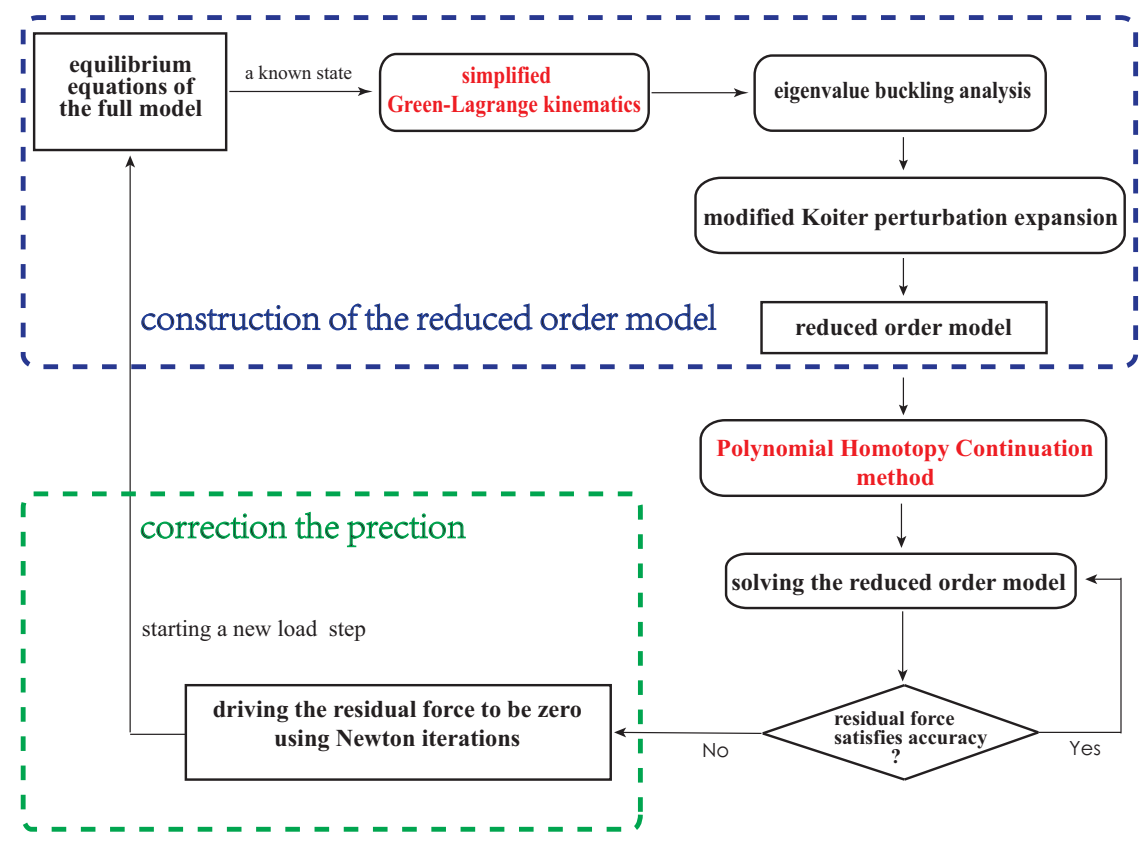

Figure 1: Flow chart of the improved Koiter-Newton method

\subsection{Solution of the reduced order model, using the PHC method}

The reduced order model (7) obtained in section 3.1 is a nonlinear system of equations with $m+1$ degrees of freedom, which could also be expressed as polynomial equations. The polynomial homotopy continuation method is a robust solver to find all the roots of a polynomial system. The basic idea is to start with known solutions of a known start system and then track those solutions as we deform the start system into the system that we wish to solve.

The PHC method operates in two stages. Firstly, homotopy methods exploit the structure of $P$ to find a root count and to construct a start system $P^{0}(x)=0$ that has exactly as many regular solutions as the root count. This start system is embedded in the homotopy, as given by:

$$
H(x, t)=\gamma(1-t) P^{0}(x)+t P(x), t \in[0,1]
$$

where $\gamma \in \mathbb{C}$ is a random number.

In the second stage, as $t$ moves from 0 to 1 , numerical continuation methods trace the paths that originate at the solutions of the start system towards the solutions of the target system.

Following the above procedures, the reduced order model is solved reliably by the polynomial homotopy continuation method, which is another optimization compared to the original Koiter-Newton method. The numerical solution is used as an initial nonlinear predictor for the response of the structure. Compared to traditional Newton methods which use a linear predictor, a significantly larger step size is obtained using the Koiter-Newton approach due to a better nonlinear predictor provided by the reduced order model, see the red, dashed curve plotted in Fig. 2. During the simulation of the reduced order model, the unbalanced force residual $\mathbf{f}_{r}(9)$ is calculated to judge whether the applied reduced order model is sufficiently accurate, as given by: 


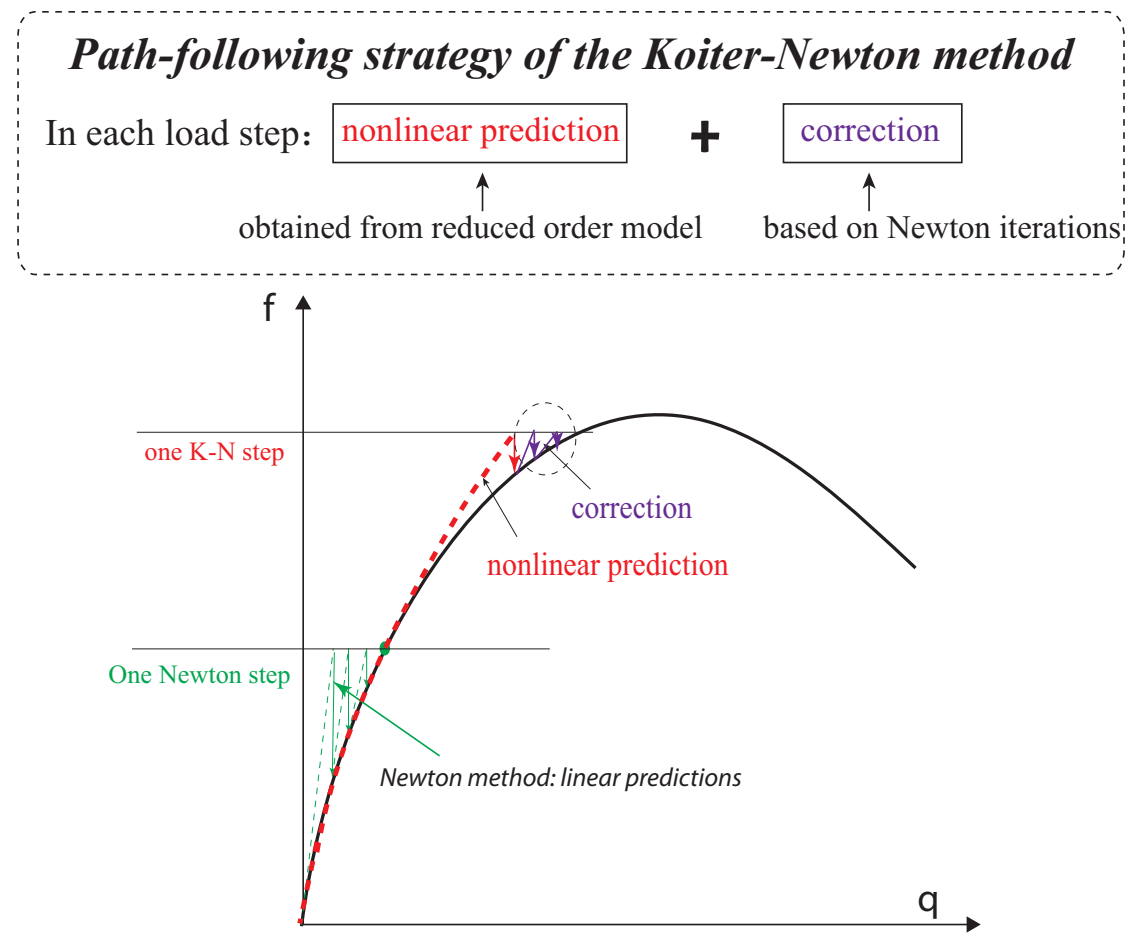

Figure 2: The path-following strategy

$$
\mathbf{f}_{r}=\lambda \mathbf{f}_{\text {ext }}-\mathbf{f}_{\text {int }},
$$

where $\mathbf{f}_{\text {int }}$ is the internal force vector of the current iteration step, $\mathbf{f}_{\text {ext }}$ is the external force vector and $\lambda$ is the current load parameter. The internal force is calculated based on the full finite element model, thus its value is reliable. The simulation of the reduced order model will be stopped for $\mathbf{f}_{r}$ being below a given threshold. In a subsequent corrector phase, the residual is driven to zero in a manner similar to the classical Newton methods, as indicated by the pink lines of Fig. 2. Then, an updated reduced order model is constructed for further load steps, based on the new found equilibrium state of the structure. At this point, one completely incremental step of the improved Koiter-Newton method is finished.

\subsection{Computational cost}

Three basic parts in each expansion step of the improved Koiter-Newton approach can be recognized: 1) construction of the reduced order model, 2) iterative solution of the reduced order model, 3) correction of the predictor produced by the reduced order model. In Fig. 3, the computational cost of each part is assessed using the number of full linear FE systems that needs to be solved, assuming that the computational cost is dominated by a factorization and not by a forward and back substitution. It can be observed in Fig. 3 that only $3 \sim 4$ linear FE systems need to be solved in each incremental step with sufficient accuracy of the reduced order model which is controlled by a chosen threshold. Then, the computational costs of the Koiter-Newton analysis and the full nonlinear analysis based on the classical Newton method can be easily compared only using the number of the incremental steps, if the number of iterations in each incremental step of the classical Newton method is also controlled to be $3 \sim 4$.

\section{Numerical examples}

The selected examples presented in this section are severe study cases for shell buckling due to their extremely sharp snap-back turning angle of the response curve. We compare the results of our method with results obtained 


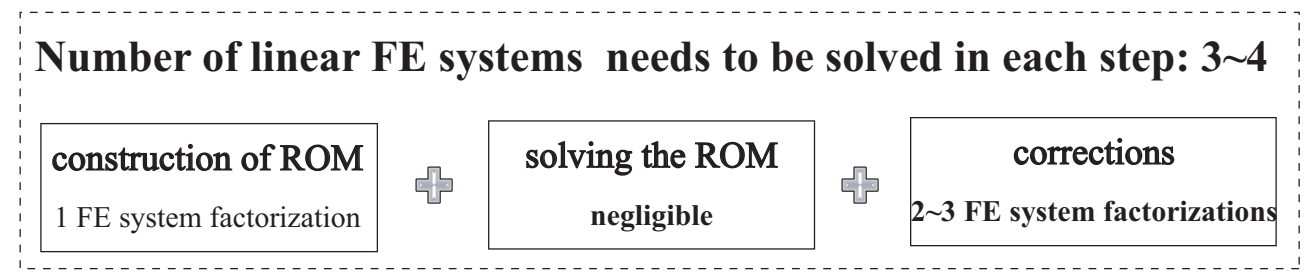

Figure 3: Computational cost in each incremental step of the improved Koiter-Newton method

from a classical Newton iteration-based nonlinear path following method which is provided in ABAQUS [22]. The ABAQUS solution uses the full set of equations of the finite element model. In addition, we also compare the results of our method with those achieved using the original Koiter-Newton method. The original Koiter-Newton method uses von Kármán kinematics to constitute the reduced order model, and adopts the classical arc-length method to solve the model.

The discrete models used in different numerical methods are equal in terms of the number of nodes and the number of elements used. As mentioned in section 3.3, the computational cost per step of the Newton method and the KoiterNewton method is of the same order, hence the number of steps needed to trace the equilibrium path are regarded sufficient for a fair comparison.

In this study, we only compare our results with the existing numerical solutions. The comparison with experimental results is difficult, since current numerical model does not consider the material degenerations, realistic boundary conditions and realistic imperfections.

\subsection{Laminated composite cylinder, $z 15$}

A classical laminated composite cylinder, named z15 cylinder, has been analyzed by Degenhardt [23] using the traditional Newton path-following method. The sketch of the cylinder is given in Fig. 4. A non-symmetric stacking sequence $\left[24^{\circ},-24^{\circ}, 41^{\circ},-41^{\circ}\right]$ is considered with orthotropic material properties $E_{11}=157362 \mathrm{~N} / \mathrm{mm}^{2} ; E_{22}=$ $10092 \mathrm{~N} / \mathrm{mm}^{2} ; G_{12}=5321 \mathrm{~N} / \mathrm{mm}^{2} ; v_{12}=0.277 ; \rho=1600 \mathrm{Kg} / \mathrm{mm}^{3}$. The cylinder is fully clamped at the bottom and fixed along the top rim but can move in axial direction in which a uniform compression load is applied.

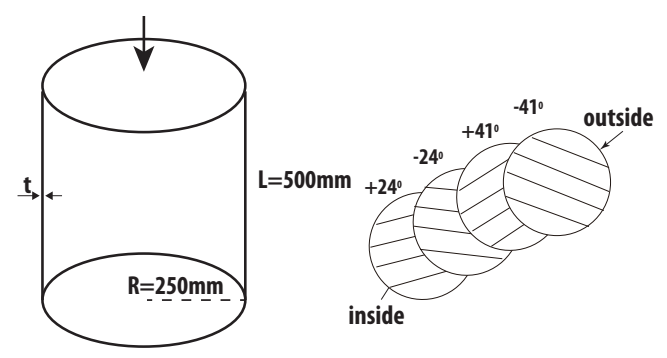

Figure 4: The laminated composite cylinder

We start with a convergence study to derive a suitable mesh size providing sufficient accuracy for the later nonlinear structural analysis. The first bifurcation load obtained from the eigenvalue analysis are used as a reference in the convergence study. Results of the convergence study are depicted in Fig. 5. It can be concluded that the improved Koiter-Newton method converges at a better rate since more accurate strain measure was used. A comparison of the linear buckling mode with the analysis results obtained from ABAQUS and the original Koiter-Newton method reveals a good match, as shown in Fig. 6, using a mesh size 10mm.

We perform a nonlinear structural analysis for the perfect cylinder geometry to obtain a reference result for a plausibility check. There are, in total, 15 DOFs in the reduced order model, where the first degree of freedom is related to the primary path, and the others are related to the 14 closely-spaced buckling modes. The response curves 


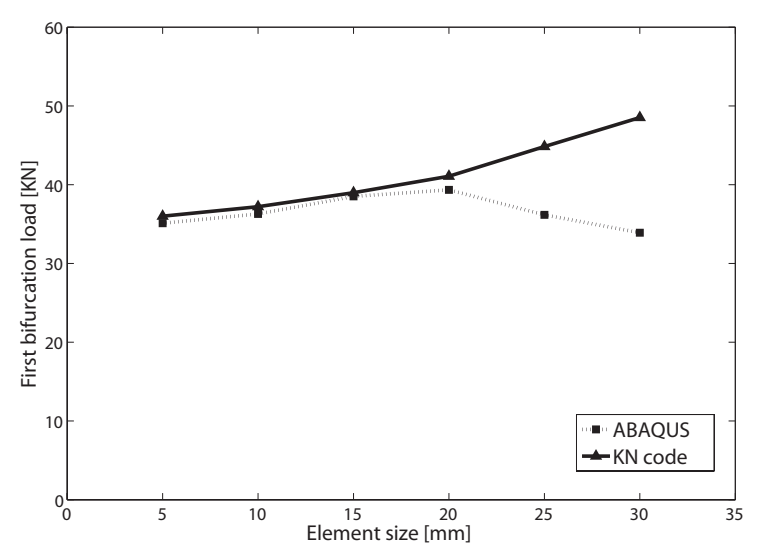

(a) Original Koiter-Newton method

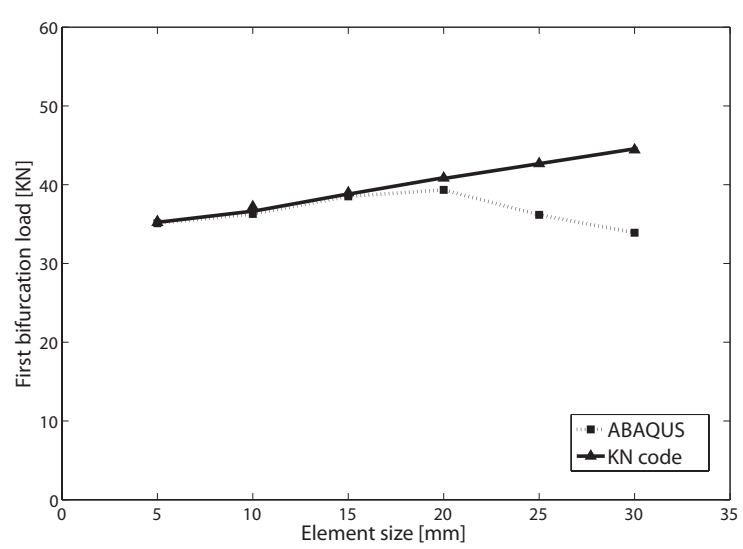

(b) Improved Koiter-Newton method

Figure 5: Convergence study of the cylinder

about the end-shortening are plotted in Fig. 7. The original Koiter-Newton method meets convergency difficulties to trace response curve after the limit point. However, the response curve obtained using the improved Koiter-Newton method reveals a better match with the ABAQUS result, not only near the limit point but also in the post-buckling stage. The complete response can be obtained if more Koiter-Newton steps are carried out in the post-buckling path. ABAQUS based on the Newton method adopts 69 steps to trace the response curve shown in Fig. 7, while the improved Koiter-Newton method only uses 3 steps. As mentioned in sec. 3.3, the computational cost in one step of the Newton method and the Koiter-Newton method is roughly the same.

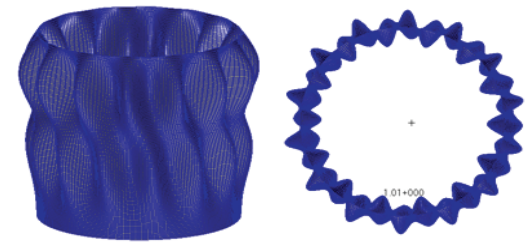

ABAQUS

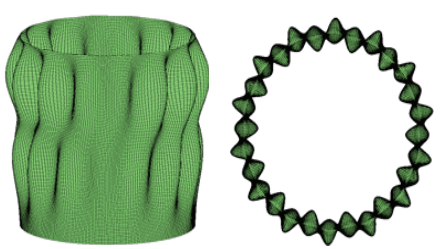

original KN method

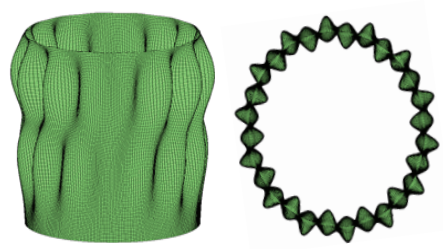

improved KN method

Figure 6: Comparison of the first buckling modes obtained using different methods

One laterally centralized load is applied in the middle $(1 / 2 \mathrm{~L})$ of the cylinder surface to model the geometric dimple imperfection. There are, in total, 16 degrees of freedom in the reduced order model for imperfection analysis. The first degree of freedom is related to the primary path, the second degree of freedom to the lateral load, and the others are related to the 14 closely-spaced buckling modes. The nonlinear buckling analyses for different values of the lateral load are carried out using the improved Koiter-Newton method. In Fig. 8, the limit loads of the various perturbation loads are plotted as a knock-down factor curve and compared to the results obtained from ABAQUS and the original Koiter-Newton method. It can be seen that when the lateral load is not very large, smaller than $2.5 \mathrm{~N}$, the values of buckling loads match very well with the results produced using ABAQUS, while the error becomes large in the descent range of the knock-down factor curve and seems to be small again when the lateral load is large enough. The reason is that the Koiter asymptotic analysis is only numerically valid under a small imperfection amplitude. However, it can be concluded in Fig. 8 that the improved Koiter-Newton method reveals a better performance compared to the original method.

The computational cost of the imperfection analyses using the Koiter-Newton approach is compared with results obtained using ABAQUS. To obtain the knock-down factor curve shown in Fig. 8, ABAQUS needs to recalculate the 


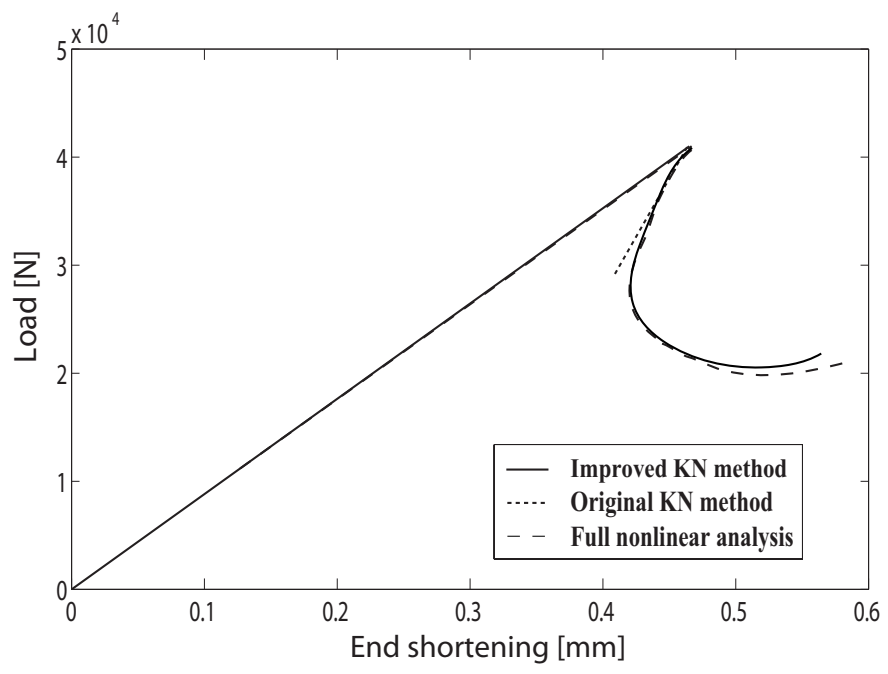

Figure 7: Response curves of the perfect cylinder

entire FE model 9 times and each time it needs approximate 57 incremental steps in average, that is ABAQUS needs in total $57 \times 9$ incremental steps. However, since the reduced order model does not need to be reconstructed for different lateral loads, the Koiter-Newton approach only needs 1 incremental step to construct the reduced order model once for the perfect cylinder. This demonstrates again that the Koiter-Newton approach is much more computational efficient if used for imperfection analyses.

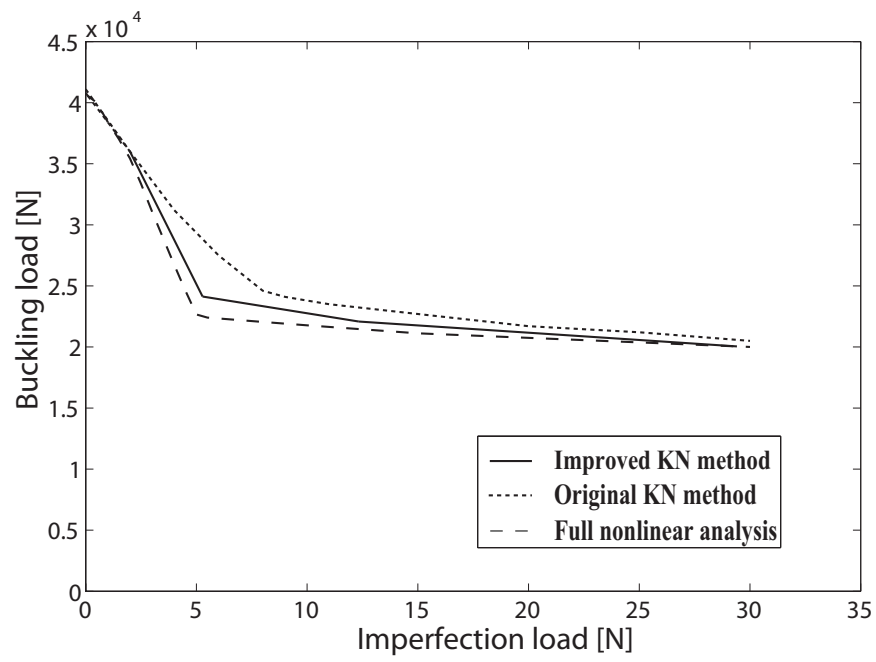

Figure 8: Knock-down curves obtained from imperfection-sensitivity analyses

\subsection{Isotropic cone}

An isotropic conical shell under axial compression in Fig. 9 has been analyzed before by Rahman and Jansen [16], with material properties $E=56950 \mathrm{~N} / \mathrm{mm}^{2}$ and $v=0.3$. The "MSS4" boundary condition [16] is applied. One end of the conical shell is fixed and an axial load was applied at the other end. All the displacement degrees of freedoms are restrained at the edge of the fixed end while the rotational degrees of freedoms remain free. At the loaded end the 
displacements are fixed in radial direction. We constrain those axial displacements such that they remain the same at all nodes of the edge and thereby eliminating the possibility of warping at the edge.

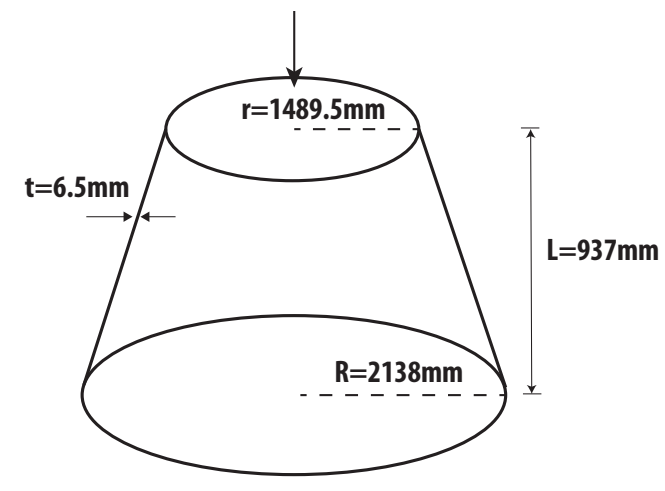

Figure 9: The isotropic cone

In Table 1, the first bifurcation load achieved using the proposed method is compared with those obtained using the original KN method, the finite element method in DIANA study [16] and the semi-analytical tools BAAC [24], respectively. The number ' $N$ ' in Table 1 denotes the number of circumferential full waves. In Fig. 10 we show the first buckling mode and the first and second order displacement fields provided by the improved Koiter-Newton method. It can be seen that the first order displacement field indicates the deformations of the axial compression, and the second order displacement field is similar to the first buckling mode.

Table 1: Comparison of the first bifurcation load [N].

\begin{tabular}{lccccc}
\hline $\mathrm{N}$ & Elements & Improved KN method & Original KN method & DIANA & BAAC \\
\hline 10 & $120 \times 36$ & $9.0147 \times 10^{6}$ & $9.0223 \times 10^{6}$ & $9.1071 \times 10^{6}$ & $9.0142 \times 10^{6}$ \\
\hline
\end{tabular}

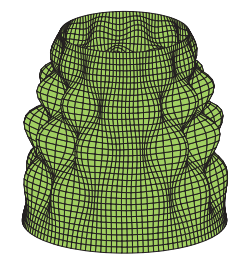

First buckling mode

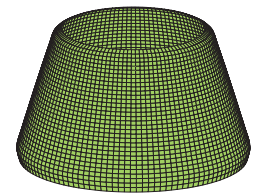

First order field

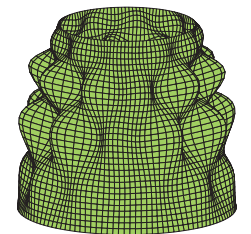

Second order field

Figure 10: Deformations obtained using the improved Koiter-Newton method

A geometric imperfection of the shape of the first buckling mode with an amplitude of $10 \%$ of the shell thickness has been used for the analysis of the imperfect structure. Here, the buckling modes are scaled such that the maximum out of plane displacement corresponds to the shell thickness. The Koiter-Newton method requires only the first buckling mode to construct the reduced order model. In general, clustered buckling modes are a common case for cylindrical and conical shells and the construction of a corresponding reduced order model which accurately captures the post-buckling response requires several modes as demonstrated in the previous example. However, the use of the first buckling mode as a suitable imperfection shape triggers the post-buckling deformation and thus a single mode analysis is possible. The end-shortening curves of the imperfect cone are compared in Fig. 11. The original Koiter-Newton method dose not converge in the post-buckling stage, thus it can obtain response of the structure only satisfactorily up to the buckling load. However, the response curve obtained using the improved method matches very well with that calculated by the full nonlinear analysis. The complete response can be obtained if more Koiter-Newton 
steps are carried out in the post-buckling path. The improved Koiter-Newton analysis requires three KN steps whereas the full Newton iteration-based nonlinear reference analysis needs 63 steps.

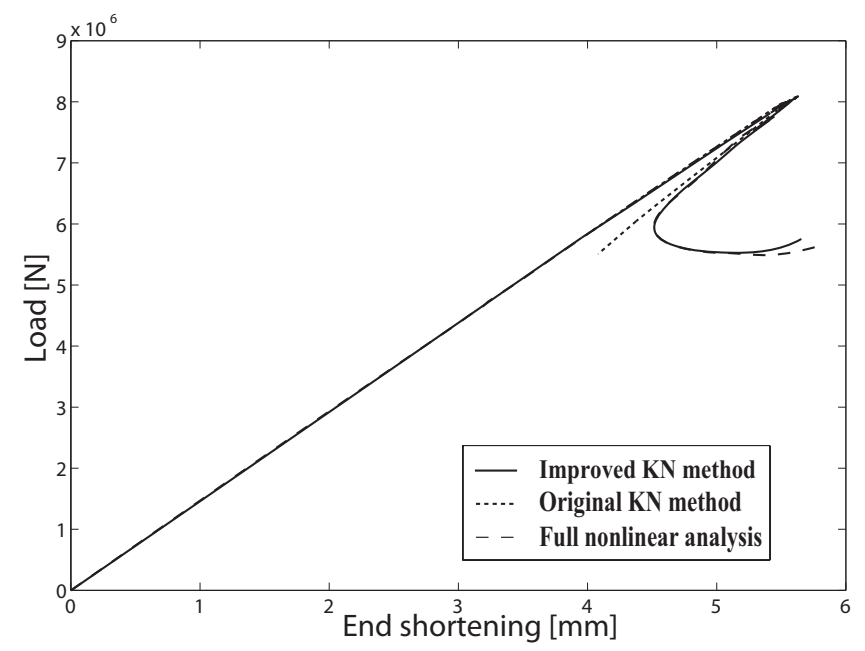

Figure 11: Response curves of the imperfect cone

\subsection{Stiffened cylinder}

An isotropic stiffened cylinder specified in Table 2, which has been studied by Liang [3] using ABAQUS, are reanalyzed using the improved Koiter-Newton method. In work [3], it was found that the results obtained from the stringer shell model correspond well with results of an alternative smeared stiffener model. For the stringer shell model, all the stiffeners inside the cylindrical shell are modeled using shell elements. In this study we use a smeared stiffener concept. The stiffeners were not modeled explicitly but taken into account with a general shell stiffness contribution (10) which was assigned to the cylinder in the constitutive equations [25].

Table 2: Configuration of the stiffened cylinder, [MPa, mm]

\begin{tabular}{ccc}
\hline Material & Cylinder dimensions & Stiffener dimensions \\
\hline E: 70000 & Radius: 400 & Height: 5.2 \\
$v: 0.34$ & Length: 1000 & Thickness: 0.55 \\
& Thickness: 0.55 & Number of stiffeners: 90 \\
\hline
\end{tabular}

$\mathrm{K}_{\text {smeared }}=\left[\begin{array}{cccccc}53569.1 & 14801 & 0 & 28855.8 & 0 & 0 \\ 14801 & 43532.3 & 0 & 0 & 0 & 0 \\ 0 & 0 & 14365.7 & 0 & 0 & 0 \\ 28855.8 & 0 & 0 & 106673.9 & 373.1 & 0 \\ 0 & 0 & 0 & 373.1 & 1097.4 & 0 \\ 0 & 0 & 0 & 0 & 0 & 456.5\end{array}\right]$.


The results of the four different analysis models are listed in Table 3. The first buckling modes obtained from the smeared stiffener models using ABAQUS and the Koiter-Newton methods, respectively, are shown in Fig. 12. The mode has 11 full waves in circumferential direction and one full wave in axial direction.

Table 3: Comparison of the first buckling load

\begin{tabular}{ccccc}
\hline Methods \& models & $\begin{array}{c}\text { ABAQUS } \\
\text { (stringer model) }\end{array}$ & $\begin{array}{c}\text { ABAQUS } \\
\text { (smeared model) }\end{array}$ & $\begin{array}{c}\text { Original KN } \\
\text { (smeared model) }\end{array}$ & $\begin{array}{c}\text { Improved KN } \\
\text { (smeared model) }\end{array}$ \\
\hline First buckling load, [N] & $1.018 \times 10^{5}$ & $1.133 \times 10^{5}$ & $1.113 \times 10^{5}$ & $1.095 \times 10^{5}$ \\
Relative error, [\%] & None & 11.3 & 9.3 & 7.5 \\
\hline
\end{tabular}

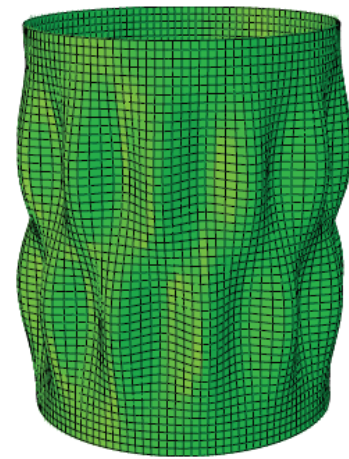

ABAQUS

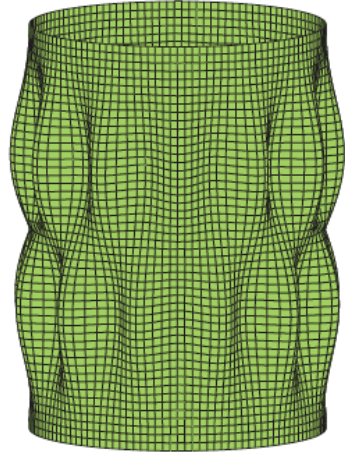

original KN method

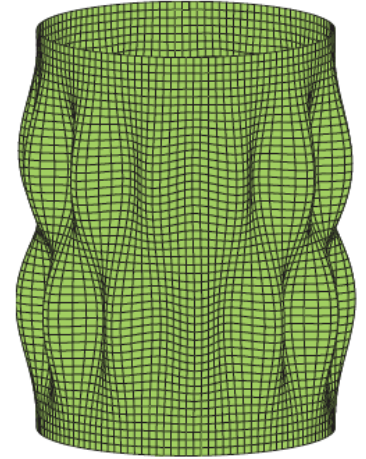

improved KN method

Figure 12: Comparison of the first buckling modes, using the smeared stiffener model

One laterally centralized load $2 \mathrm{~N}$ is applied in the middle $(1 / 2 \mathrm{~L})$ of the cylinder surface to model the geometric imperfection. There are, in total, 20 degrees of freedom in the reduced order model for imperfection analysis. The first degree of freedom is related to the primary path, the second degree of freedom to the lateral load, and the others are related to the 18 closely-spaced buckling modes. The end-shortening curves are plotted in Fig. 13. ABAQUS uses the displacement control and the damping factor in the Newton method to directly jump through the sharp turning angle near the limit point, hence the snap-back segment is lost. However, the improved Koiter-Newton method achieves the entire snap-back path of the structure, and the re-stiffness path in the post-buckling stage matches very well with the ABAQUS solution. ABAQUS based on the Newton method adopts 53 steps to trace the response curve, while the improved Koiter-Newton method only use 2 steps.

\section{Conclusions}

The conical and cylindrical shells perform an extremely sharp snap-back-turning near the limit point. Although the original Koiter-Newton method provides a more reliable path-tracing analysis near the initial post-buckling stage, compared to the traditional Newton based path-following technique, this method also meets difficulties to achieve the entire snap-back response that is far beyond the limit point. Hence, the improved Koiter-Newton method is proposed in this paper to overcome the above problem. The simplified Green-Lagrange kinematics are implemented to consider the stress redistribution properly after buckling. The polynomial homotopy continuation method is used to solve the lower-order nonlinear reduced order model reliably and efficiently. A composite cylinder, a metal cone and a stiffened cylinder are used to evaluate the path-following performance of the improved Koiter-Newton method for the snapback case. The geometric imperfections are modeled using either the linear buckling mode or the lateral load. The numerical results show that the improved method is a numerically robust, accurate and efficient path-follow technique to obtain the entire snap-back response of the structure. 


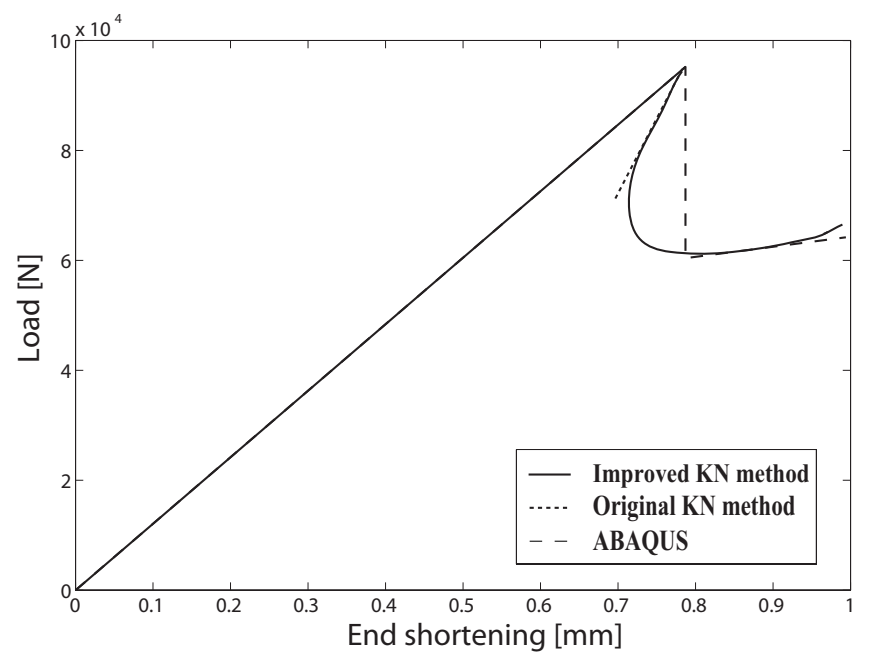

Figure 13: Response curves of the imperfect stiffened cylinder

\section{Acknowledgments}

The research leading to these results received funding from the European Community's Seventh Framework Programme ([FP7/2007-2013]) under grant agreement $n^{\circ} 282522$. This research work was also sponsored by Laboratory Independent Innovation project of Qian Xuesen Laboratory of Space Technology.

\section{References}

[1] E. Barkanov, E. Egltis, F. Almeida, M.C. Bowering, G. Watson, Optimal design of composite lateral wing upper covers. Part II: Nonlinear buckling analysis, Aerospace Science and Technology 51 (2016) 87-95.

[2] Z. Ou, X. Yao, X. Zhang, X. Fan, Buckling of a stiff thin film on a compliant substrate under anisotropic biaxial prestrain, Science China Physics, Mechanics $\mathcal{E}$ Astronomy 59(2) (2016) 624601-1-624601-10.

[3] K. Liang, Y. Zhang, Q. Sun, M. Ruess, A new robust design for imperfection sensitive stiffened cylinders used in aerospace engineering, Science China Technological Sciences 58 (2015) 1-7.

[4] J. Cai, Y. Xu, J. Feng, J. Zhang, Buckling and post-buckling of rotationally restrained columns with imperfections, Science China Physics, Mechanics $\mathcal{E}$ Astronomy 55(8) (2016) 1519-1522.

[5] S. G. P. Castro, R. Zimmermann, M. A. Arbelo, R. Khakimova, M. W. Hilburger, R. Degenhardt, Geometric imperfections and lower-bound methods used to calculate knock-down factors for axially compressed composite cylindrical shells, Thin-Walled Structures 74 (2014) 118132.

[6] K. Liang, M. Ruess, M. Abdalla, An eigenanalysis-based bifurcation indicator proposed in the framework of a reduced-order modeling technique for non-linear structural analysis, International Journal of Non-Linear Mechanics 81 (2016) 129-138.

[7] S. A. Ragon, Z. Gürdal, L. T. Watson, A comparison of three algorithms for tracing nonlinear equilibrium paths of structural systems, International Journal of Solids and Structures 139 (2002) 689-698.

[8] S. E. Leon, E. N. Lages, C. N. D. Araujo, G. H. Paulino, On the effect of constraint parameters on the generalized displacement control method, Mechanics Research Communications 56(56) (2014) 123-129.

[9] C. Bisagni, Composite cylindrical shells under static and dynamic axial loading: An experimental campaign, Progress in Aerospace Sciences 78 (2015) 107-115.

[10] L. Friedrich, T. Schmid-Fuertes, K. Schröder, Comparison of theoretical approaches to account for geometrical imperfections of unstiffened isotropic thin walled cylindrical shell structures under axial compression, Thin-Walled Structures 40(7) (2015) 1-9.

[11] S. G. P. Castro, C. Mittelstedt, F.A.C. Monteiro, R. Degenhardt, Evaluation of non-linear buckling loads of geometrically imperfect composite cylinders and cones with the Ritz method, Composite Structures 122(122) (2015) 284-299.

[12] K. Liang, M. Abdalla and Z. Gürdal, A Koiter-Newton approach for nonlinear structural analysis, International Journal for Numerical Methods in Engineering 96(12) (2013) 763-786.

[13] K. Liang, M. Ruess and M. Abdalla, The Koiter-Newton approach using von Kármán kinematics for buckling analyses of imperfection sensitive structures, Computer Methods in Applied Mechanics and Engineering 279 (2014) 440-468.

[14] W. T. Koiter, On the stability of the elastic equilibrium, Delft University of Technology, PhD thesis (1945).

[15] T. Rahman, E. L. Jansen, Finite element based coupled mode initial post-buckling analysis of a composite cylindrical shell, Thin-Walled Structures 48 (2010) 25-32. 
[16] T. Rahman and E. L. Jansen, Finite element based initial post-buckling analysis of shells of revolution, in 49th AIAA/ASME/ASCE/AHS/ASC Structures, Structural Dynamics and Materials Conference, Schaumburg, Illinois, US (2008), pp. 1-11.

[17] D. Robert, et al, Concepts and Applications of Finite Element Analysis, 4th Edition. (Wiley,2001).

[18] T. Y. Li, Numerical solution of multivariate polynomial systems by homotopy continuation methods, Acta Numerica 6 (1997) $399-436$.

[19] A. Morgan, A. Sommese, Computing all solutions to polynomial systems using homotopy continuation, Applied Mathematics and Computation 24(2) (1992) 115-138.

[20] K. Liang, M. Ruess, M. Abdalla, Co-rotational finite element formulation used in the Koiter-Newton method for nonlinear buckling analyses, Finite Elements in Analysis and Design 116 (2016) 38-54.

[21] Y. Zhou, I. Stanciulescu, T. Eason, M. Spottswood, Nonlinear elastic buckling and postbuckling analysis of cylindrical panels, Finite Elements in Analysis and Design 96 (2015) 41-50.

[22] ABAQUS: Theory manual. Providence, R.I.: Hibbitt, Karlsson and Sorensen (1992).

[23] R. Degenhardt, A. Kling, A. Bethge, J. Orf, L. Kärger, R. Zimmermann, K. Rohwer, A. Calvi, Investigations on imperfection sensitivity and deduction of improved knock-down factors for unstiffened CFRP cylindrical shells, Composite Structures 92(8) (2010) 1939-1946.

[24] G. Zhang, Stability Analysis of Anisotropic Conical Shells, Delft University of Technology, PhD thesis (1993).

[25] I. Elishakov, J. Arbocz, C. D. Babcock jr, A. Libai, Buckling of Structures: Theory and Experiment. Elsevier Science Publishers B.V., Amsterdam (1988). 